\title{
Integration of an Earth-Based Science Team during Human Exploration of Mars
}

\author{
Steven P. Chappell \\ KBRwyle \\ KBRwyle/HAC/37C \\ 2400 NASA Parkway \\ Houston, TX 77058 \\ steven.p.chappell@nasa.gov \\ Trevor G. Graff \\ Jacobs Engineering Group \\ Science Department \\ 2224 Bay Area Blvd. \\ Houston, TX 77058 \\ trevor.g.graff@nasa.gov
}

\author{
Kara H. Beaton \\ KBRwyle \\ KBRwyle/HAC/37C \\ 2400 NASA Parkway \\ Houston, TX 77058 \\ kara.h.beaton@nasa.gov
}

\author{
Kelsey E. Young \\ UTEP/Jacobs JETS Contract at NASA \\ 2101 NASA Parkway \\ Houston, TX, 77058 \\ kelsey.e.young@nasa.gov
}

\author{
Carolyn Newton \\ University of North Dakota \\ Department of Space Studies \\ Clifford Hall Room 512 \\ 4149 University Ave. Stop 9008 \\ Grand Forks, ND 58202 \\ carolynnewton12@gmail.com \\ David Coan \\ Stinger Ghaffarian Technologies \\ 2101 NASA Parkway \\ Houston, TX 77058 \\ david.coan@nasa.gov
}

\author{
Andrew F. J. Abercromby \\ NASA Johnson Space Center \\ 2101 NASA Parkway \\ Houston, TX 77058 \\ andrew.abercromby@nasa.gov
}

\author{
Michael L. Gernhardt \\ NASA Johnson Space Center \\ 2101 NASA Parkway \\ Houston, TX 77058 \\ michael.l.gernhardt@nasa.gov
}

\begin{abstract}
NASA Extreme Environment Mission Operations (NEEMO) is an underwater spaceflight analog that allows a true mission-like operational environment and uses buoyancy effects and added weight to simulate different gravity levels. A mission was undertaken in 2016, NEEMO 21, at the Aquarius undersea research habitat. During the mission, the effects of varied operations concepts with representative communication latencies associated with Mars missions were studied. Six subjects were weighed out to simulate partial gravity and evaluated different operations concepts for integration and management of a simulated Earth-based science team (ST) who provided input and direction during exploration activities. Exploration traverses were planned in advance based on precursor data collected. Subjects completed science-related tasks including presampling surveys and marine-science-based sampling during saturation dives up to 4 hours in duration that simulated extravehicular activity (EVA) on Mars. A communication latency of 15 minutes in each direction between space and ground was simulated throughout the EVAs. Objective data included task completion times, total EVA time, crew idle time, translation time, ST assimilation time (defined as time available for the science team to discuss, to review and act upon data/imagery after they have been collected and transmitted to the ground). Subjective data included acceptability, simulation quality, capability assessment ratings, and comments. In addition, comments from both the crew and the ST were captured during the post-mission debrief. Here, we focus on the acceptability of the operations concepts studied and the capabilities most enhancing or enabling in the operations concept. The importance and challenges of designing EVA timelines to account for the length of the task, level of interaction with the ground that is required/desired, and communication latency, are discussed.
\end{abstract}

\section{TABLE OF CONTENTS}

1. INTRODUCTION ....................................................1

2. RESEARCH QUESTIONS..................................2

3. STUDY DESIGN \& METHODS ...........................2

4. RESULTS................................................................3

5. DISCUSSION.......................................................6

6. CONCLUSIONS .....................................................8

REFERENCES.......................................................9

BIOGRAPHY ..................................................99

ACKNOWLEDGEMENTS....................................11

\section{INTRODUCTION}

The NASA Extreme Environment Mission Operations (NEEMO) Project conducts analog missions that send groups of astronauts, engineers, and scientists to live in the Florida International University's (FIU) Aquarius Reef Base, an underwater habitat. Aquarius is the world's only undersea research facility and is located approximately $5.6 \mathrm{~km}(3.5$ miles) off the coast of Key Largo, FL at a depth of 19 meters (62 feet). NASA and the NEEMO project have used the Aquarius facility since 2001 . The habitat and its surroundings provide a high fidelity analog for space exploration. Living and working in the undersea environment allows participants (referred to as "aquanauts") to experience some of the same challenges that will be found on future exploration missions to distant asteroids, moons, or planets (e.g., Mars). The aquanauts are able to simulate living in a spacecraft and test extravehicular activity (EVA) techniques and exploration concepts for future space missions. The underwater environment has the benefit of enabling the aquanauts to simulate different 
gravity levels through the calculated addition of weights or buoyant floats to aquanauts during excursion dives outside of the habitat. On shore, mission control facilities allow streaming of audio, video, and data from the crew inside the habitat as well as while outside the habitat performing simulated EVAs; similarly, communication streams flow from mission control to the habitat. Latency can be introduced into the 2way audio, video, and data streams to simulate the light-time delays in communication that will occur when humans venture into deep space. As an example, destinations such as the Mars surface would introduce communication latencies with Earth from 4-22 minutes in each direction, depending on planetary alignments. The NEEMO mission discussed in this paper simulated concepts for human exploration of Mars systems aligned with NASA's Evolvable Mars Campaign (EMC) [1-4]. NEEMO 21 also evaluated science operations that informed the development of NASA's exploration EVA operations concepts.

The paper will address the communication latency-related EVA research conducted during the NEEMO 21 mission that took place in July 2016 with a duration of 16 days. The mission had a split format with 2 crew surfacing and 2 crew entering the habitat half way through while two others remained in the habitat for all 16 days; this provided 6 total aquanaut test subjects for the study consisting of NASA astronauts and other engineers and scientists. Exploration traverses were executed during the mission to perform marine science tasks in the area of the habitat with a simulated communication latency of 15- minute OWLT (one-way light time) between the habitat and the shore-side support team. This communication latency was chosen to represent a long latency relevant to the Mars system and to cross-over to studies performed in other analogs.

\section{Exploration Traverse Operations Concepts}

As the OWLT increases for potential human exploration destinations such as the Mars system, achieving meaningful Earth-based science team (ST) input during an EVA will be challenging [5], including bandwidth constraints limiting the amount of data (including voice, video, still imagery, text messages, location, and scientific instrument data) that can be transmitted between space and ground [6]. Based on these challenges, one operations concept (ConOps) could implement a nearly autonomous crew to execute the science objectives with a ground-based ST acting primarily as a passive observer, who only provide opportunistic feedback across latency during the EVA as able. In this case, the ST would mainly provide strategic input in-between EVAs, as opposed to within EVAs. An alternate ConOps could implement strategically designed EVA timelines with built-in timing accommodations to allow for the crew to transmit science data to the ST, the ST to analyze and interpret this data (during the available time frame defined as the "ST assimilation time") and send guidance and direction for subsequent EVA tasks to the crew during the EVA. This ConOps does not preclude the first ConOps, but also adds the opportunity for tactical (i.e., intra-EVA) ST input to actively influence timeline execution.
It also has the benefit of not having to revisit exploration sites on different days to achieve science input and thus affording more opportunity to explore new sites. Both ConOps offer scientific and operational advantages and were employed at NEEMO 21, optimized to the specific marine science objectives for a given EVA.

Mars mission ConOps, capabilities, and communication protocols have been tested and iteratively developed during previous analog tests. They include the results and lessons learned from previous analog tests, beginning with NASA's Desert Research and Technology Studies (DRATS, 2010-11) $[7,8]$ and continuing through the Pavilion Lake Research Project (PLRP, 2011-14) [9], NASA Extreme Environment Mission Operations (NEEMO) missions 16-20 (2012-16) $[10,11]$, and BASALT (2015-present) [12].

\section{RESEARCH QUESTIONS}

The NEEMO 21 EVA research questions addressed in this paper were focused on assessing ConOps and capabilities for enabling meaningful space-ground interactions during an EVA in the presence of communication latency. They can be summarized as:

1. Do Mars-mission ConOps, capabilities, and communications protocols, strategically designed to include ST assimilation time within an EVA, work acceptably (from a science and operations perspective) for 15-minute OWLT latency? What improvements are desired, warranted, or required?

A. Does acceptability change based on the amount of ST assimilation time given or additional capabilities used to provide that input?

2. Do Mars-mission operations concepts, capabilities, and communications protocols designed without ST assimilation time work acceptably (from a science and operations perspective) for 15-minute OWLT latency? What improvements are desired, warranted, or required?

3. Which capabilities are enabling and significantly enhancing for the Mars-mission ConOps and protocols being tested?

To investigate these research questions, marine sciencebased exploration EVA traverses and timelines were designed in advance and executed during the NEEMO 21 mission.

\section{StUdy DESIGN \& Methods}

Our study design integrated our ConOps research questions with marine science objectives for the mission. The marinescience objectives included both new reef exploration with the intent to perform targeted sampling of specific marinescience species as well as revisiting of marine-science sites sampled during NEEMO 20 [11]. While performing new reef exploration, timelines were strategically designed to include different amounts of ST assimilation time within and across EVAs. This was achieved through having the crew perform 
presampling surveys, having a ST assess the information from those surveys to prioritize sampling (while an independent EVA task was being performed), and then following up at a later time with the crew performing sampling based on ST prioritization.

To address research questions 1 and $1 \mathrm{~A}$, the mission EVA schedule was designed such that there were 3 EVAs during which presampling surveys and sampling were performed within the same EVA, each with different amounts of ST assimilation time between the 2 phases (named the Intra-EVA condition). There were also 3 instances of the separation of presampling and sampling phases by 1-2 days (named the Inter-EVA condition) (see Figure 2 for EVA layout across days, ST assimilation time and sampling statistics). To address research question 2, during the portions of the mission in which follow-up science was performed at NEEMO 20 science sites, the presampling phase was considered to have been performed during NEEMO 20 and thus only resampling at those sites based on predefined ST products created to guide the crew was necessary. This required that the crew be more autonomous and only receive feedback from the ST after sampling had already begun. This condition was referred to as "Sampling-Only". For all of these conditions, the ST was led by NASA planetary scientists, and included FIU marine scientists that utilized the samples being taken by the crew for separate independent research.

Throughout all EVAs, the crew and ST had the opportunity for continuous (delayed) communication regarding the marine science being performed. To facilitate those interactions, capabilities were implemented based on the lessons learned from previous analogs that have defined a baseline from which to continue to test and iterate. The core capabilities to facilitate interactions included:

- From the two extravehicular (EV) crewmembers (2) to the intravehicular (IV) crewmember (1) and groundbased ST:

- Streaming video from EV crew helmet cameras realtime to IV and delayed to the ST;

- Streaming audio from the EV crew real-time to IV and delayed to the ST;

- Data from scientific instruments used by the EV crew (verbally communicated and recorded by IV in a science data tool visible over delay by the ST, with the ST also able to hear the delayed audio; see below);

- Imagery taken by the EV crew (imagery was not transmitted during the EVAs; rather the images were downloaded post-EVA and made available to IV and the ST).

- Between the IV crewmember and the ST and Mission Support Center (MSC):

- Delayed audio communications;

- Delayed text/data via the Playbook Mission Log [13];

- A tactical EVA management tool (TEMT) used by the IV crewmember to guide EV task sequencing and to record actual task durations (the MSC could view a delayed screen capture of the TEMT); the tool also provided the capability to project future tasks start times based on being ahead or behind on the timeline and determine EVA time remaining;

- A science data tool was provided by the ST before each EVA and the IV crew used it to direct the EV crew and to record presampling and sampling data during the science operations; the tool was visible to the ST through delayed screen sharing.

\section{Subjective Data Collection}

During the mission, the study team consistently applied a set of field-tested evaluation techniques that use surveys of acceptability, capability assessment, and simulation quality ratings [7, 8, 10-12, 14-17]. The surveys included individual and consensus ratings by the EV crew, IV crew, and ST team. Initial ratings and associated recommendations were recorded individually by team-members. Overall consensus ratings and recommendations were then discussed and agreed upon by crewmembers and, separately, by the ST team in postEVA consensus meetings. An additional opportunity to review, discuss, and finalize consensus ratings and comments was provided at a post-mission debrief to maximize consistency across all study conditions.

\section{Objective Timing Data Collection}

Detailed timing data including task durations (EV and ST), translation times, ST assimilation time, and crew idle time (for the purposes of addressing our research questions this is defined as any crew idle time directly attributable to waiting on ST input), were collected to investigate correlations between subjective ratings and objective task and ConOps performance.

\section{RESULTS}

ST Assimilation Time: All EVAs were executed according to the study design to include varied amounts of ST assimilation time based on the duration of independent EVA tasks being performed between presampling and sampling. Intra-EVA presampling and sampling were performed on mission day (MD) 6, 7, and 14 and Inter-EVA presampling and sampling were paired on MD6/7, MD 10/12, and MD 11/13; Figure 2 shows the layout of the Intra-EVA, Inter-EVA, and SamplingOnly conditions across the mission. Figure 1 focuses on the Intra-EVA condition. MDs 6 and 7 were planned to start with presampling followed by an independent task and finishing with sampling based on ST input received. With a 15-minute OWLT latency, the ST had to send initial ST-sampling input prior to the times depicted by the black diamonds for it to be received before sampling. In actuality, on both MD 6 and 7 the ST sent sampling input substantially before it was needed (28 minutes early on MD 6 and 1 hour and 8 minutes early on MD 7). MD 14 was planned as a 3-hour and 25-minute time block with no predetermined duration for the presampling and sampling phases. In this case, a dynamic leaderboard approach was used in which 3 sampling priority 
inputs/updates were sent by the ST, 2 before the start of sampling, and 1 after.

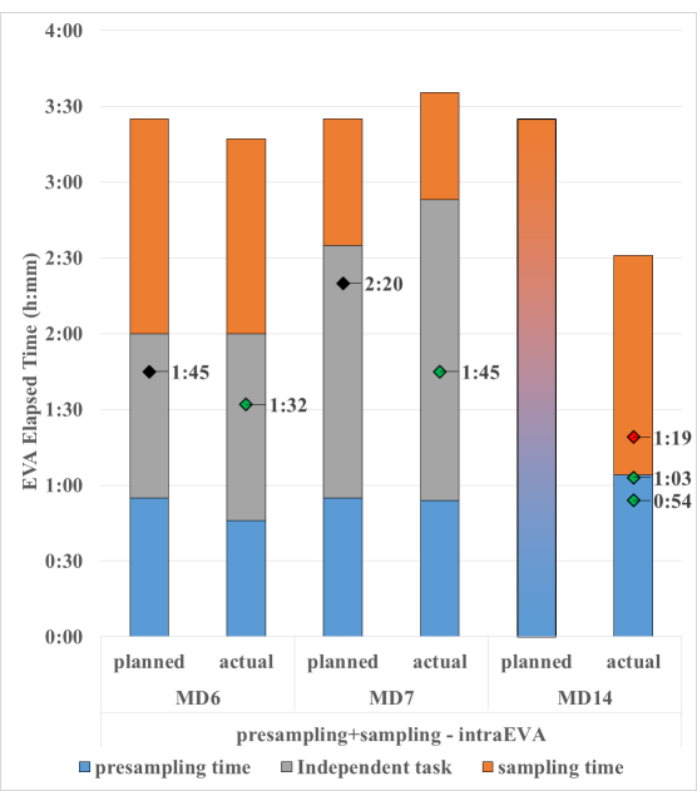

Figure 1. Presampling and sampling task sequencing and duration for Intra-EVA condition; planned and actual task durations shown as well as planned task separation. Also shown are planned no-later-than ST input times (black diamonds) and actual ST input times (green/red diamonds).

Figure 2 also shows for each presampling phase the number of candidate-sample locations identified by the EV crew and thus the number of candidate samples that needed to be assessed and prioritized by the ST. In addition, for each sampling phase, the number of samples requested by the ST and taken by the EV crew are shown. For the Intra-EVA ConOp, all ST assimilation was performed while the ST was also monitoring other activities (e.g. independent tasks that still required ST attention, such as pre-sampling for another site or sampling of a NEEMO 20 site). For the Inter-EVA ConOp, the ST monitored the presampling phase and created a prioritized list of samples to be taken during the EVA. Post-EVA, imagery taken during the EVA was transmitted to the ST and reviewed (estimated at 30 seconds per candidate sample) to determine if revisions should be made to the sampling priorities (which happened $10 \%-15 \%$ of the time); the imagery was also used to create annotated sampling guidance that was provided to the crew for the sampling phase via in-water electronic cue cards. Creation of the cue cards took approximately 2 minutes per sample. Figure 2 shows for the sampling - InterEVAs on MDs 7, 12 and 13 estimated ST assimilation time ("calc. ST assim. time") based on the timing estimates for image review and creation of sampling guidance cue card input. Figure 2 also shows there was a wide variation in the average time that the crew took to identify candidate sample locations and the average time to perform a sample.

Table 1 shows the time spent on subtasks within the presampling and sampling phases; mean durations and standard deviations are shown for each subtask for each ConOp. The largest percentage of time during the presampling phase was spent on candidate-sample location search, observation (i.e., contextual description, sample description, providing helmet camera shots of candidates), and instrument utilization (i.e., using instruments on each candidate sample). These subtasks provided the information needed by the ST to assess which candidate-sample locations should be sampled. During the sampling phase, substantial time was spent in site set up, additional observation, sample collection, sample preservation, and site cleanup. A higher percentage of presampling phase time was spent searching for candidate sample locations in the Inter-EVA ConOps than for intra-EVA.

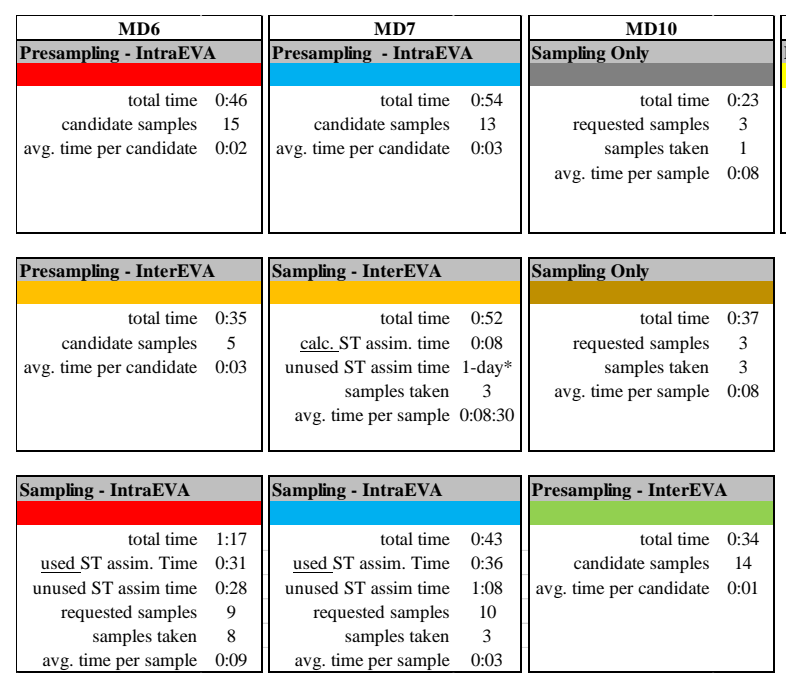

Figure 2. Presampling and sampling phase statistics for all EVs; times in hr:mm; color coding indicates matched pairs of pre-sampling and sampling; *number of days separation between matched pairs, not all of which was available. 
Table 1. EV crew presampling and sampling task duration as a function of ConOps (means and standard deviations in $\mathrm{mm}: \mathrm{ss})$.

\begin{tabular}{|c|c|c|c|c|c|c|}
\hline \multirow[b]{2}{*}{ Presampling Tasks } & \multicolumn{3}{|c|}{$\begin{array}{l}\text { Intra } E V A \\
\text { Condition }\end{array}$} & \multicolumn{3}{|c|}{$\begin{array}{l}\text { Inter } E V A \\
\text { Condition }\end{array}$} \\
\hline & $\begin{array}{c}\text { Mean } \\
\text { Dura- } \\
\text { tion }\end{array}$ & \pm & $\begin{array}{c}\text { St. } \\
\text { Dev. }\end{array}$ & $\begin{array}{c}\text { Mean } \\
\text { Dura- } \\
\text { tion }\end{array}$ & \pm & $\begin{array}{c}\text { St. } \\
\text { Dev. }\end{array}$ \\
\hline Candidate Sample Search & $11: 30$ & \pm & $02: 16$ & $19: 32$ & \pm & $13: 51$ \\
\hline Observation & $14: 30$ & \pm & 03:20 & 09:42 & \pm & $06: 12$ \\
\hline Temporary Tag Search & $02: 40$ & \pm & 00:51 & $08: 05$ & \pm & 06:01 \\
\hline Instrument Utilization & $10: 20$ & \pm & $01: 42$ & 08:39 & \pm & 07:40 \\
\hline Navigation/Translation & 04:20 & \pm & $02: 24$ & 03:06 & \pm & $01: 44$ \\
\hline Site Clean-Up & $03: 40$ & \pm & $00: 14$ & $03: 25$ & \pm & $01: 58$ \\
\hline Other & 01:30 & \pm & 01:05 & $06: 27$ & \pm & 06:03 \\
\hline
\end{tabular}

\begin{tabular}{|c|c|c|c|c|c|c|c|c|c|}
\hline \multirow[b]{2}{*}{ Sampling Tasks } & \multicolumn{3}{|c|}{$\begin{array}{l}\text { Intra EVA } \\
\text { Condition }\end{array}$} & \multicolumn{3}{|c|}{$\begin{array}{l}\text { Inter EVA } \\
\text { Condition }\end{array}$} & \multicolumn{3}{|c|}{$\begin{array}{l}\text { Sampling-Only } \\
\text { Condition }\end{array}$} \\
\hline & $\begin{array}{c}\text { Mean } \\
\text { Dura- } \\
\text { tion }\end{array}$ & \pm & $\begin{array}{c}\text { St. } \\
\text { Dev. }\end{array}$ & $\begin{array}{c}\text { Mean } \\
\text { Dura- } \\
\text { tion }\end{array}$ & \pm & $\begin{array}{c}\text { St. } \\
\text { Dev. }\end{array}$ & $\begin{array}{c}\text { Mean } \\
\text { Dura- } \\
\text { tion }\end{array}$ & \pm & $\begin{array}{c}\text { St. } \\
\text { Dev. }\end{array}$ \\
\hline Site Set-Up & 07:34 & \pm & $01: 50$ & 07:20 & \pm & 01:56 & $04: 15$ & \pm & $00: 15$ \\
\hline Temporary/Permanent Tag Search & 05:56 & \pm & 00:04 & 09:20 & \pm & $06: 21$ & 08:00 & \pm & $06: 00$ \\
\hline Observation & $16: 52$ & \pm & $08: 36$ & $19: 20$ & \pm & $12: 08$ & $02: 15$ & \pm & $01: 45$ \\
\hline Sample Collection & $14: 12$ & \pm & $07: 27$ & 19:10 & \pm & $15: 06$ & 05:30 & \pm & 03:30 \\
\hline Sample Preservation & 09:28 & \pm & $06: 47$ & 07:40 & \pm & 04:33 & 01:30 & \pm & 00:00 \\
\hline Navigation/Translation & $05: 14$ & \pm & 02:59 & $06: 50$ & \pm & $03: 24$ & $00: 45$ & \pm & $00: 15$ \\
\hline Site Clean-Up & $15: 52$ & \pm & $07: 36$ & $11: 40$ & \pm & $05: 33$ & $02: 45$ & \pm & 00:15 \\
\hline Other & 09:27 & \pm & $02: 33$ & $16: 10$ & \pm & 08:34 & 05:30 & \pm & 01:30 \\
\hline Unknown (Due to Communication & & & & & & & & & \\
\hline Dropouts) & $0: 11: 47$ & \pm & 0:00:47 & $0: 50: 30$ & \pm & $0: 21: 30$ & 0:04:15 & \pm & $0: 00: 15$ \\
\hline
\end{tabular}

\section{Acceptability Ratings}

Figure 3 shows the consensus EV/IV crew and ST acceptability ratings. Overall, the Intra-EVA ConOps was rated borderline (5: Improvements warranted) by both the crew and the ST; the Inter-EVA ConOps was rated acceptable (4: Minor improvements desired) by both.

The Intra-EVA ConOps was rated more poorly than InterEVA mainly due to higher workload and schedule pressure within an EVA to both identify and take samples. There was a higher workload on the ST who had to both monitor ongoing tasks and at the same time formulate sampling priorities. The workload was higher on the IV crewmember as well, who had to manage ongoing real-time EV tasks at the same time as interacting with the ST across delay regarding their input in regards to sampling. However, minimal training time was available for the crew and ST, which contributed to the challenge of the flight control tempo during this ConOps. In the Inter-EVA ConOps, the ST could take additional time to review imagery taken during the EVA (which could not be transmitted real-time during the EVA) and formulate cue cards to aid as a sampling guide for the EV/IV crew. In addition, the EV/IV crew could take time to review the sampling cue cards and increase efficiency by formulating a sampling plan.

The Sampling-Only ConOps was rated as acceptable by the ST mainly due to the ability to create detailed sampling plans in advance based on prior mission data and little additional intra-EVA input was required from the ST for sampling success. In all ConOps, the crew identified a key improvement being the ability to use a navigation system to reliably locate previously visited candidate sampling locations (from presampling in the Intra-EVA or Inter-EVA ConOps) or new sampling locations. The as-executed navigation capability often included still-frames taken from the crew's video, compiled by the ST in between EVAs that attempted to enable the crew to follow visual cues real-time to return to a sampling location. An improvement in this capability was found to be more important for the Inter-EVA ConOps than for the IntraEVA ConOps as more time had passed since visiting candidate-sample locations; it was deemed particularly important 
for the Sampling-Only ConOps (visiting sites from previous missions) as none of the current mission's crew had been to those locations.

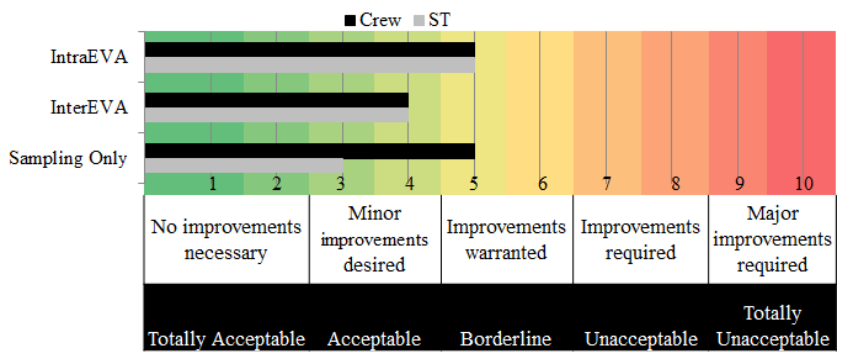

\section{from the EV/IV crew and the ST.}

Capability Assessment Ratings: Figure 4 shows the collected capability assessment ratings from the EV/IV crew and the ST. All capabilities assessed were rated as essential/enabling or significantly enhancing by both the crew and the ST except for the ability to have 2 IV crewmembers. The crew noted that 2 IV crewmembers would not be necessary (except in the case of emergencies) with the improvements identified in other capabilities, and additional training and experience.

Figure 3. Operations Concept Acceptability Ratings

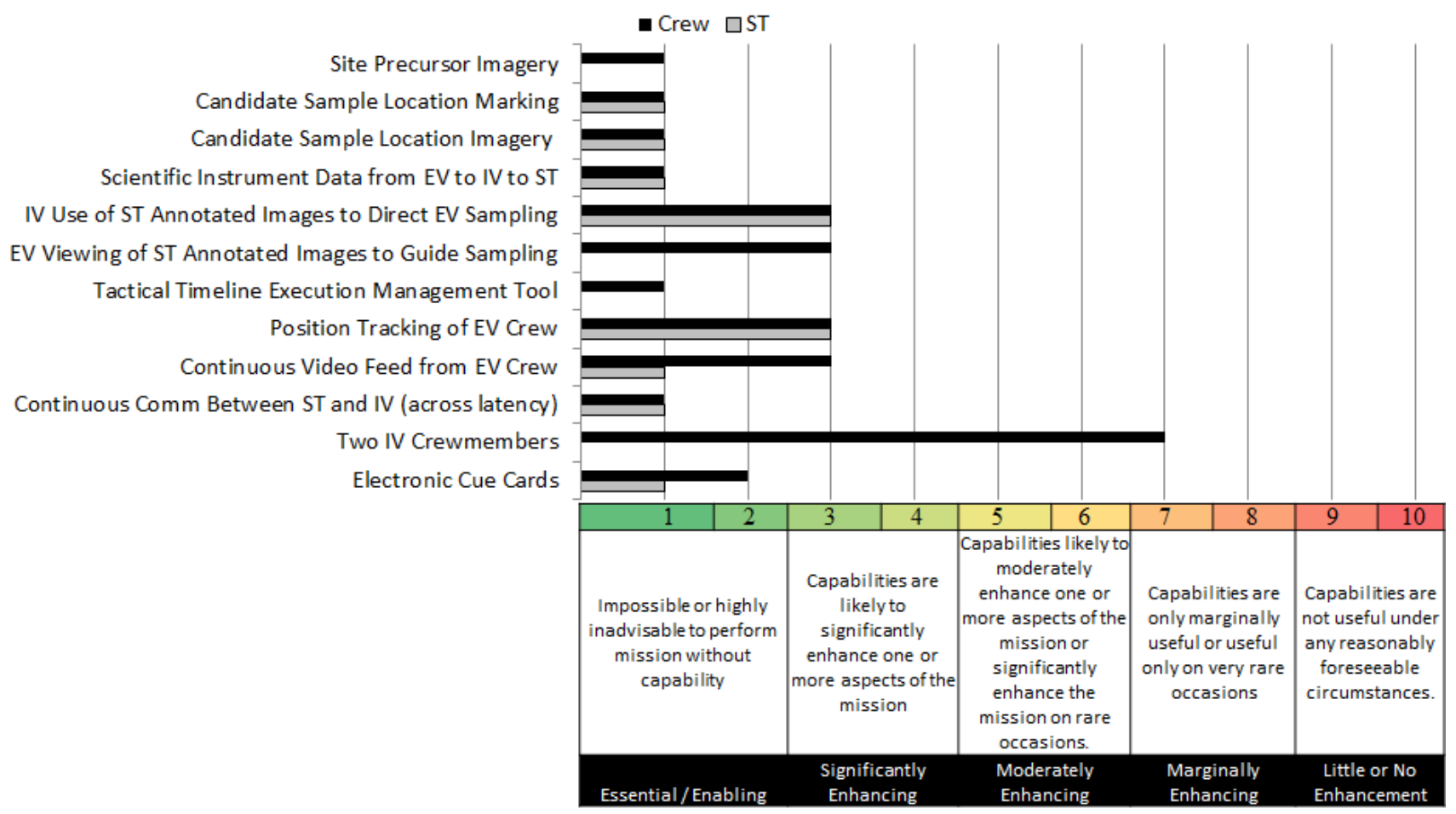

Figure 4. Consensus capability assessment ratings from the EV/IV crew and the ST.

\section{DISCUSSION}

\section{Research Question 1: ConOps Acceptability}

Mars-mission ConOps, capabilities, and communications protocols developed and tested during previous analog tests strategically designed to include additional ST assimilation time were rated as borderline acceptable from a science and operations perspective for 15-minute OWLT latency. Summary-level improvements desired, warranted, or required include:

- The ability to use a navigation system to electronically mark candidate samples, relocate candidate samples and to track crew position in relation to a planned traverse is assumed to be a capability we will have when we go to Mars, even if there is not a GPS-like system. This navigation and position tracking would be visible by EV, IV, and MSC. However, it should be noted that during NASA's 2009 DRATS test 100 m root mean square error in position was found to be totally acceptable to find individual rocks that the crew had not previously visited [15]. The terrain during DRATS 2009 was relatively open (i.e., Black Point lava flow in Arizona) whereas the topography around Aquarius consists of coral spurs and grooves in which it is more difficult to get context. Thus the requirements for position accuracy may be terraincomplexity dependent. The level of accuracy desired due to terrain complexity during this mission may not be necessary for all areas on Mars.

- Direct transmission of scientific instrument data (e.g. spectral data) from EV to IV and ST would have increased efficiency and accuracy versus verbal communication and manual recording of data. The inability to 
transmit instrument data real time was an artifact of the analog environment and could have been viewed as inadequate simulation quality; however, in this case the crew regarded this as an identified improvement.

- In regards to IV use of ST annotated images to direct EV sampling during an EVA, higher resolution imagery (than achievable by screen captures of low-resolution video as was done during this mission) transmitted during the EVA would have improved the ability to discern the intent of the ST. The required "pixels on target" necessary to discern whether the correct science target had been achieved was adequate most of the time but as we stated in the results section, higher resolution imagery provided post-EVA did alter science decisions 10-15\% of the time in the Inter-EVA condition. It should be noted that more "pixels on target" can be achieved either with higher resolution video or imagery or by decreasing the distance from the camera to the target.

- Improving the efficiency of annotated image creation was deemed important as well as improvements in the efficiency of the transmission of annotated images via the Mission Log. Image annotation was accomplished during this mission through a process that involved taking a screen capture from video, importing that screen capture into image modification software, annotation of the image, and importing of the image into the Mission Log for transmission; this process took multiple minutes depending on the complexity of the annotation. Improvements in efficiency that would allow for capture, annotation, and transmission within the same software would likely make the process take less than a minute and thereby provide more time to be focusing $100 \%$ on ongoing crew tasks.

- EV viewing of ST annotated images to guide sampling was not available for this mission but the crew felt the ability for EV crew to receive data from the IV and the ST would have been an improvement (capability assessment rating = 3: significantly enhancing). Having the EV crew be able to view images directly in the Mission Log would have eliminated the need for IV to interpret the images received and verbally communicate the guidance to the EV crew; this could generally mean saving a few minutes or possibly even make it possible to provide the ST guidance where it would not be possible to effectively provide verbally.

- A tactical EVA management tool for the IV crew to manage the EVA (e.g. direct task sequencing, monitor compliance with planned task duration, project future task start times based on as-run timeline) was found to be very important (capability assessment rating $=1$ : essentiallenabling). However the as-tested version of this tool did not provide the capability to reorder and add new tasks which could be an important aspect of future EVAs in non-engineered environments such as Mars.

- For the first use of electronic cue cards for the EV crew at a NEEMO mission, the cue cards were made comprehensive, including all detailed procedures and content required for the entire mission. Improvements noted by the
EV crew were in the electronic cue card user interface to allow for simpler navigation to desired content and focusing of the content available to the crew on what is need for each individual EVA.

\section{Research Question 1A: ST Assimilation Time Effects}

Acceptability of the Mars-mission baseline ConOps is acceptable based on giving the ST "extra time" in the Inter-EVA condition to provide input to the crew, along with additional data (i.e., high-resolution imagery) to be used to provide that input. It should be noted that ST assimilation time was not controlled during any condition. The mean ST assimilation time in the Intra-EVA condition was $\sim 31 \mathrm{~min}( \pm \sim 4$ min st. dev.) while in the Inter-EVA condition it was calculated to be $\sim 24 \mathrm{~min}( \pm \sim 15 \mathrm{~min}$ st. dev.). These ST assimilation times are comparable to each other and do not show that more time was spent in the Inter-EVA condition that might account for the more acceptable rating than the Intra-EVA condition. The increase in acceptability was due to the tools and number of personnel available to the ST making the workload higher in the Intra-EVA condition. The workload was higher due to the fact that the limited personnel in the ST had to be focused on ongoing EVA tasks as well as on formulating sampling input. In the Inter-EVA condition, the ST could focus solely on the presampling phase and make any final adjustments in their sampling guidance post-EVA.

The ST used high-resolution still imagery of candidate samples post-EVA in the Inter-EVA condition (as opposed to low-resolution video only during the EVA for the Intra-EVA condition) to refine their sampling priorities in approximately $10 \%-15 \%$ of the cases and created annotated imagery to guide sampling. However, as is seen in figure 2, there was unused ST assimilation time (mean $\sim 48 \mathrm{~min} \pm 20 \mathrm{~min}$ st. dev.) in the Intra-EVA condition; thus image review and annotation could have been accomplished within an EVA with the capability for image transmission (or more "pixels on target" with video) and enough ST personnel to perform the required tasks.

\section{Research Question 2: ConOps without ST Assimilation Time}

The Mars-mission ConOps, capabilities, and communications protocols designed without ST assimilation time (as in the Sampling-Only condition, visiting previous mission sites with pre-EVA provided sampling guidance) were considered acceptable overall, from both a science and operations perspective, for 15-minute OWLT latency. However, there is substantial recognition that the success of EVA tasks and timelines without ST assimilation time are highly dependent on crew training and their ability to work as an EV/IV team independent from ST input, other than the sampling plans provided in advance of the EVA.

\section{Research Question 3: Capabilities Assessment}

Capabilities rated as essential/enabling or significantly enhancing for the Mars-mission ConOps and protocols tested were (ratings in parentheses): 
- Site precursor imagery (1: essential/enabling): imagery sufficient to use in planning of exploration regions by the ST and for EV crew visual navigation when approaching regions;

- Candidate-sample location marking (1: essential/enabling): use of a marker by the EV crew to unambiguously mark candidate samples in video and imagery that are sent to IV and the ST;

- Candidate-sample location imagery (1: essential/enabling): images and/or video screen captures of candidate samples that contain the candidate-sample location markers for reference;

- Scientific instrument data from EV to IV to ST (1: essential/enabling): transmission of data on candidate samples taken by scientific instruments to IV and the ST rather than transcription of EV audio by IV into a science data tool;

- IV use of ST annotated images to direct EV sampling (3: significantly enhancing): ST or IV annotation of images captured from video or images sent from the crew containing information to guide sampling by EV;

- EV viewing of ST annotated images to guide sampling (3: significantly enhancing): while this capability was not tested at N21, the crew noted that the ability for EV crew to view annotated imagery sent by IV or the ST would be significantly enhancing;

- Tactical EVA management tool (1: essential/enabling): a tool that provides a means to display and track EVA task sequences, task durations, projects forward the impacts of finishing tasks ahead or behind on the overall timeline, and provides key timers that help the crew track when they should expect input from the ST on STdependent tasks;

- Position tracking of EV crew (3: significantly enhancing): tracking of the crews position in relation to planned traverses and known landmarks (e.g. terrain, habitat);

- Continuous video feed from EV crew (1: essential/enabling): the ability for the ST and IV to see a continuous video feed from both EV crew members;

- Continuous communication between ST and IV (1: essential/enabling): the ability to have continuous communication throughout the entire EVA (i.e. no gaps in communication coverage);

- Electronic cue cards (1: essential/enabling): an electronic means of displaying maps, EVA timeline tasks/sequencing, summary-level procedures, and other information relevant to each EVA.

\section{Modeling for Integration of Analog ConOps Testing}

A substantial number of analog studies have investigated different ConOps for conducing Mars-relevant science, matching the strengths of the particular analog environment with scientific and operationally relevant research objectives desired to be addressed. The effects of different communication latencies, bandwidth and capability limitations, number and distribution of personnel, and other operational parameters have been examined, which has enabled us to define our baseline ConOps and understand how operational efficiency and the opportunity for scientific productivity is affected as various operational parameters change. In general, results across many analog missions have demonstrated that the operational and scientific acceptability of a given ConOp vary with communication latency and bandwidth limitations. While these, and other, findings have helped shape a basic understanding of human planetary exploration science operations under certain test conditions, logistical, temporal, and budgetary limitations preclude our ability to conduct individual field tests to investigate all possible combinations of ConOps, latencies, bandwidth constraints, science task types, etc. However, the results already obtained from prior analog studies can form the basis of a parametric model that could extend the results of previous field tests and strategically inform which operational scenarios would benefit most directly from additional field tests. Such a model could also help determine which ConOps are most appropriate for different science objectives and could assist in understanding how multiple ConOps may be necessary within a mission, for each EVA, and possibly within an EVA. This model would also provide input to other modeling and testing efforts, such as those called out in the Integrated EVA Human Research Plan [18], which identifies the need for the development of an EVA Human Health and Performance (HHP) parametric model that combines EVA task and ConOps information from analogs with results from partial gravity human physiological testing in EVA suits to predict HHP for humans on the Mars surface [19].

\section{Conclusions}

In summary, there was not an advantage to the Inter-EVA ConOps over the Intra-EVA ConOp that could not have been obtained through provision of real-time image transfer (or more "pixels on target" with video) during the EVAs and additional personnel on the ST to process and annotate imagery. Improvements in the imagery tools and the other capability improvements identified would reduce the ST and IV workload and potentially make the Intra-EVA ConOp more subjectively acceptable. It should also be noted that a possible limitation in this investigation was that the nature of the marine-science tasks for this mission, while requiring targeting of specific marine-science species for sampling, were driven to achieve large numbers of samples and this may have affected the results in the Intra- vs Inter-EVA comparison (i.e., achieving sampling numbers in some cases was more important than continuing to identify new available candidate samples). Crew, MSC and ST training on the science objectives, operations objectives and the software and hardware tools available are critical to the successful execution of the Intra-EVA ConOps, where the lack of necessary timing and clarity of interactions between the crew and ground can directly affect EVA productivity and crew idle time. In the Sampling-Only ConOps, EV/IV training and electronic cue cards with clarity of ST intent becomes more paramount. Each ConOps tested provides advantages and disadvantages and it is likely that each will be used during the exploration of Mars. The choice of ConOps for Mars EVAs will likely be dependent on the science objectives of that EVA balanced with the operational costs. Future work, such as that being 
carried out in the BASALT (Biologic Analog Science Associated with Lava Terrains) Research Project [12], will continue to refine and test these ConOps and capabilities while performing real, non-simulated science and thus better prepare us for human exploration of Mars.

\section{REFERENCES}

[1] A. F. Abercromby, S. Chappell, D. Lee, A. Howe, and M. Gernhardt, "Human Exploration of Phobos," in Proceedings of the IEEE Aerospace Conference, Big Sky, MT, 2015.

[2] D. A. Craig, P. Troutman, and N. Herrmann, "Pioneering Space Through the Evolvable Mars Campaign," in AIAA SPACE 2015 Conference and Exposition, 2015, p. 4409.

[3] D. A. Craig, N. B. Herrmann, and P. A. Troutman, "The Evolvable Mars Campaign-study status," in Aerospace Conference, 2015 IEEE, 2015, pp. 1-14.

[4] M. L. Gernhardt, A. F. Abercromby, O. S. Bekdash, S. P. Chappell, Z. Q. Li, K. H. Beaton, et al., "Human exploration missions to phobos prior to crewed mars surface missions," in Aerospace Conference, 2016 IEEE, 2016, pp. 1-20.

[5] S. G. Love and M. L. Reagan, "Delayed voice communication," Acta Astronautica, vol. 91, pp. 8995, 10// 2013.

[6] S. N. Rader, M. L. Reagan, B. Janoiko, and J. E. Johnson, "Human-in-the-loop operations over time delay: NASA analog missions lessons learned," in Proceedings of the 43rd International Conference on Environmental Systems, 2013, pp. 1-6.

[7] A. F. Abercromby, M. L. Gernhardt, and J. Jadwick, "Evaluation of dual multi-mission space exploration vehicle operations during simulated planetary surface exploration," Acta Astronautica, vol. 90, pp. 203-214, 2013.

[8] A. F. Abercromby, S. P. Chappell, and M. L. Gernhardt, "Desert RATS 2011: Human and robotic exploration of near-Earth asteroids," Acta Astronautica, vol. 91, pp. 34-48, 2013.

[9] D. S. S. Lim, A. Brady, A. Abercromby, D. Andersen, M. Andersen, R. Arnold, et al., "A historical overview of the Pavilion Lake Research Project-Analog science and exploration in an underwater environment," Geological Society of America Special Papers, vol. 483, pp. 85-115, 2011.

[10] S. P. Chappell, A. F. Abercromby, M. L. Reagan, and M. Gernhardt, "NEEMO 16: Evaluation of techniques and equipment for human exploration of near-Earth asteroids," presented at the 44th Lunar and Planetary Science Conference, The Woodlands, TX, 2013.

[11] S. P. Chappell, K. H. Beaton, M. J. Miller, T. G. Graff, A. F. Abercromby, M. L. Gernhardt, et al., "NEEMO 18-20: Analog Testing for Mitigation of Communication Latency During Human Space Exploration," presented at the IEEE Aerospace Confernce, Big Sky, MT, 2016.
[12] K. H. Beaton, S. Chappell, M. J. Miller, D. S. S Lim, and A. Abercromby, "Extravehicular Activity Operations Concepts under Communication Latency and Bandwidth Constraints," presented at the IEEE Aerospace Conference, Big Sky, MT, 2017.

[13] S. Hashemi and S. Hillenius, "Building Next Generation User Experiences@ NASA," 2014.

[14] A. F. Abercromby, M. L. Gernhardt, and H. L. Litaker, "Desert Research and Technology Studies (DRATS) 2008 evaluation of Small Pressurized Rover and unpressurized rover prototype vehicles in a lunar analog environment," NASA Johnson Space Center, Houston, TX NASA-TP-2010-216136, 2010.

[15] A. F. Abercromby, M. L. Gernhardt, and H. L. Litaker Jr, "Desert Research and Technology Studies (DRATS) 2009: Evaluation of small pressurized rover and unpressurized rover prototype vehicles in a lunar analog environment," NASA Johnson Space Center, Houston, TX TP-2012217360, 2012.

[16] A. F. Abercromby, S. P. Chappell, H. L. Litaker, M. L. Reagan, and M. Gernhardt, "NASA Research and Technology Studies (RATS) 2012: Evaluation of Human and Robotic Systems for Exploration of Near-Earth Asteroids," presented at the 44th Lunar and Planetary Science Conference, The Woodlands, TX, 2013.

[17] M. J. Miller, D. S. Lim, A. Brady, Z. Cardman, E. Bell, B. Garry, et al., "PLRP-3: Operational Perspectives of Conducting Science-Driven Extravehicular Activity with Communications Latency," 2016.

[18] A. Abercromby, S. Cupples, S. Rajulu, J. Buffington, J. Norcross, and S. Chappell, "Integrated Extravehicular Activity (EVA) Human Research Plan: 2016," 2015.

[19] A. Abercromby, J. Norcross, and S. Jarvis, "EVA Health and Human Performance Benchmarking Study," presented at the 2016 Human Research Program Investigators' Workshop, Galveston, TX, 2016.

\section{BIOGRAPHY}

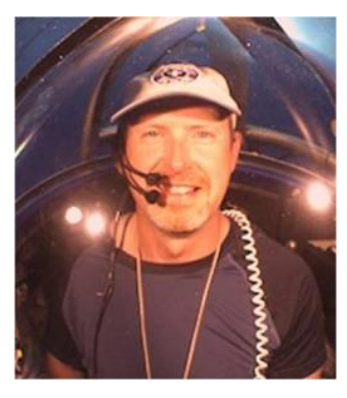

Steve Chappell attended the University of Michigan and earned a bachelor's degree in aerospace engineering. He also earned masters and doctoral degrees from the University of Colorado in aerospace engineering sciences, researching human performance and spacesuit systems in simulated reduced gravity. His career has spanned many areas of engineering and science, including work on embedded software for fighter aircraft, satellite ground systems development, and Earth-observing satellites systems engineering. In recent years, in addition to helping 
lead the Mars Moons Human Spaceflight Architecture Team, his work focused on optimizing human and system performance for the next generation of space exploration. He has extensive experience leading and taking part in research in multiple exploration analog environments including arctic, desert, underwater, alpine, and partial gravity simulators. He currently is the science operations research co-lead for the BASALT analog research project.

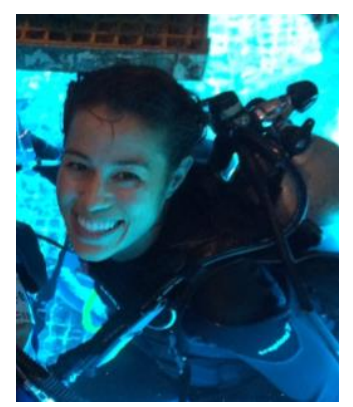

Kara Beaton received her bachelor's and master's degrees in aerospace engineering from the University of Illinois and Massachusetts Institute of Technology, respectively. She completed her doctoral studies in biomedical engineering at the Johns Hopkins University School of Medicine. She has extensive experience with operationally driven aerospace and biomedical research with the US Navy, NASA, and various clinical laboratories. She is currently a research engineer in the EVA Physiology Laboratory at JSC, where she is involved in Exploration Analogs and Mission Development, co-leading the science operations research for BASALT. She has also served on the Mars Moons Human Spaceflight Architecture team.

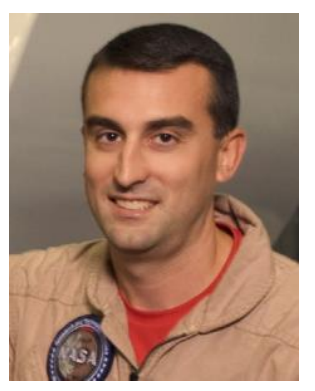

Trevor Graff is the Chief Scientist for Jacobs in the Astromaterials Research and Exploration Science (ARES) Division at NASA's Johnson Space Center. He is also the Project Manager of the Advanced Exploration Group within the Jacobs Science Department and manages the Spectroscopy and Magnetics Laboratory which conducts detailed analysis of astromaterials and planetary analogues to interpret data from various planetary landers, rovers, and orbiting spacecraft. He received a bachelor's degree in Geology and Earth Sciences from Youngstown State University in 1999, and a master's degree in Geological Sciences from Arizona State University in 2003. He was the Payload Uplink Lead for the Miniature Thermal Emission Spectrometers aboard NASA's Mars Exploration Rovers. Additionally, Trevor has served over 16 years in the United States Army.

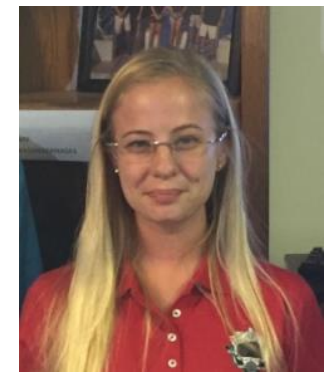

Carolyn Newton holds a B. S. in Human Factors from Embry-Riddle Aeronautical University in Daytona Beach, FL. After graduation, Carolyn worked as a training consultant at Quantum Improvements Consulting, LLC. She also completed an internship at the Space and Naval Warfare Systems Command (SPAWAR) in San Diego, as a human factors engineer/user-centered designer. During her time as an undergrad she collaborated with 2 teams of students, serving as an executive officer on 2 rotations of student expeditions to the Mars Desert Research Station. Carolyn observed mission operations and communication delays during a NASA Extreme Environment Mission Operations (NEEMO) rotation in Fall 2014 and took part in data collection in summer 2016.

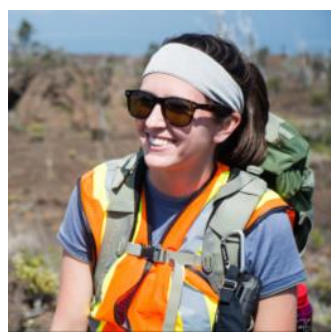

Kelsey Young is an Exploration Scientist for University of Texas, El Paso and Jacobs in the Astromaterials Research and Exploration Science (ARES) Division at NASA's Johnson Space Center. She received a B.S. in Environmental Geosciences from the University of Notre Dame and an M.S. and Ph.D. in Geological Sciences from Arizona State University. Her work focuses on conducting geologic fieldwork at sites analogous to other planetary surfaces as well as integrating science and science operations into analog missions like NEEMO. She also has extensive experience integrating field portable instruments into terrestrial field campaigns and operational concepts for planetary exploration.

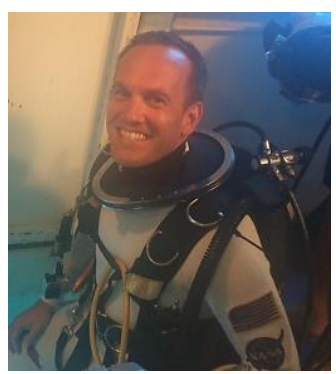

David Coan is an EVA Operations Officer and an Exploration EVA Systems Engineer, with extensive flight operations and integrated operational field testing (analog) experience. For over 20 years he has supported multiple groups at NASA's Johnson Space Center, including working in engineering, safety, and operations, and interfacing with payloads, robotics, EVA, and astronauts. He currently works in the EVA Office, developing the strategic architecture for future Exploration missions and leads integrated development testing. He is an active EVA instructor and flight controller in the Flight Operations Directoratehe plans, trains, and directs spacewalks from Mission Control - and he has led both Space Shuttle and ISS EVA operations. Coan acted as an EVA operations expert and topside support diver for NEEMO 15-21, and was an aquanaut crewmember on NEEMO 20. He co-led EVA operations for PLRP 2014, was a RATS 2012 crewmember, and he performed as a Traverse Director for the Desert RATS 2009 and 
2010 missions. He received a bachelor's degree in mechanical engineering from Texas A\&M University.

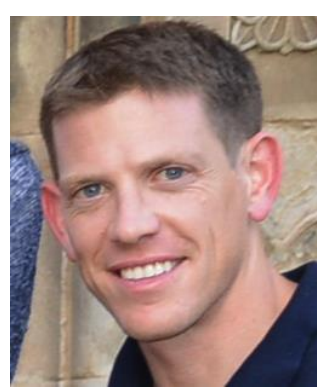

Andrew Abercromby received an M.Eng. in mechanical engineering from the University of Edinburgh in 2002 during which he worked on $X-38$ in the Flight Mechanics Laboratory at Johnson Space Center (JSC). He earned a Ph.D. in motor control from the University of Houston while working in the JSC Neurosciences Laboratory and is now the lead of NASA's EVA Physiology Laboratory and also serves as EVA Scientist for the Biomedical Research and Environmental Sciences Division. His current research focuses on measurement and optimization of human performance and operations in extreme exploration environments and includes research studies in desert, ocean, lake, virtual reality, Arctic, and Antarctic environments including experience in saturation and under-ice scientific research diving.

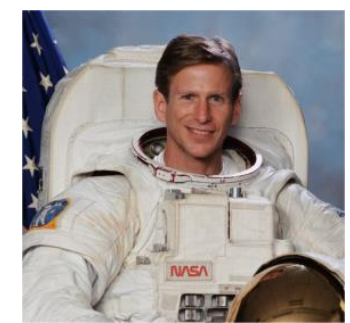

Michael Gernhardt is a NASA astronaut who has been a mission specialist on four Space Shuttle missions. He has a bachelor's degree in physics from Vanderbilt University as well as master's and doctorate in bioengineering from the University of Pennsylvania. He is the manager of the NASA Johnson Space Center EVA Physiology Laboratory, project lead for the EAMD team, and the lead for the Mars Moons Human Spaceflight Architecture Team.

\section{ACKNOWLEDGEMENTS}

The authors wish to thank and acknowledge the many scientists and engineers who contributed to the analysis described in this report. The authors also wish to thank Jennifer Tuxhorn and Jackie Reeves for their thorough reviews. 